\title{
An aging Interventions Testing Program: study design and interim report
}

Richard A. Miller, ${ }^{1}$ David E. Harrison, ${ }^{2}$ Clinton M. Astle, ${ }^{2}$ Robert A. Floyd, ${ }^{3}$ Kevin Flurkey, ${ }^{2}$ Kenneth L. Hensley, ${ }^{3}$ Martin A. Javors, ${ }^{4}$ Christiaan Leeuwenburgh, ${ }^{5}$ James F. Nelson, ${ }^{6}$ Ennio Ongini, ${ }^{7}$ Nancy L. Nadon, ${ }^{8}$ Huber R. Warner ${ }^{8}+$ and Randy Strong ${ }^{9}$

${ }^{1}$ Department of Pathology and Geriatrics Center, University of Michigan, Ann Arbor VA Medical Center, Ann Arbor, MI 48109-2200, USA

${ }^{2}$ The Jackson Laboratory, Bar Harbor, ME 04609, USA

${ }^{3}$ Oklahoma Medical Research Foundation, Oklahoma City, OK 73104, USA

${ }^{4}$ Department of Psychiatry, University of Texas Health Science Center at San Antonio, TX 78229, USA

${ }^{5}$ Department of Aging and Geriatrics, University of Florida, Gainesville, FL 32610, USA

${ }^{6}$ Department of Physiology and Barshop Center for Longevity and Aging Studies, University of Texas Health Science Center,

San Antonio, TX 78229, USA

${ }^{7} \mathrm{NicO}$ Research Institute, Milan, Italy

${ }^{8}$ Biology of Aging Program, National Institute on Aging, Bethesda, MD 20892, USA

${ }^{9}$ Geriatric Research, Education and Clinical Center and Research Service, South Texas Veterans Health Care System, San Antonio; and Department of Pharmacology, and Barshop Center for Longevity and Aging Studies at The University of Texas Health Science Center at San Antonio, TX 78229, USA

\section{Summary}

The National Institute on Aging's Interventions Testing Program (ITP) has developed a plan to evaluate agents that are considered plausible candidates for delaying rates of aging. Key features include: (i) use of genetically heterogeneous mice (a standardized four-way cross), (ii) replication at three test sites (the Jackson Laboratory, TJL; University of Michigan, UM; and University of Texas, UT), (iii) sufficient statistical power to detect $10 \%$ changes in

\section{Correspondence}

Richard A. Miller, Department of Pathology and Geriatrics Center, University of Michigan, Ann Arbor VA Medical Center Ann Arbor, MI 48109-0940, USA.

Tel.: 734936 2122; fax: 734647 9749; e-mail: millerr@umich.edu

Richard A. Miller, David E. Harrison and Randy Strong contributed equally to this study.

†Current address: University of Minnesota, College of Biological Sciences, St. Paul, MN 55108, USA.

Accepted for publication 26 April 2007 lifespan, (iv) tests for age-dependent changes in $\mathrm{T}$ cell subsets and physical activity, and (v) an annual solicitation for collaborators who wish to suggest new interventions for evaluation. Mice in the first cohort were exposed to one of four agents: aspirin, nitroflurbiprofen (NFP), 4-OH- $\alpha$-phenyl-N-tert-butyl nitrone (4-OH-PBN), or nordihydroguiaretic acid (NDGA). An interim analysis was conducted using survival data available on the date at which at least $50 \%$ of the male control mice had died at each test site. Survival of control males was significantly higher, at the interim time-point, at UM than at UT or TJL; all three sites had similar survival of control females. Males in the NDGA group had significantly improved survival $(P=0.0004)$, with significant effects noted at TJL $(P<0.01)$ and UT $(P<0.04)$. None of the other agents altered survival, although there was a suggestion $(P=0.07)$ of a beneficial effect of aspirin in males. More data will be needed to determine if any of these compounds can extend maximal lifespan, but the current data show that NDGA reduces early life mortality risks in genetically heterogeneous mice at multiple test sites.

Key words: aging; aspirin; longevity; mice; nordihydroguiaretic acid.

\section{Introduction}

An agent, taken in food or water, that could delay aging would potentially have a major effect on human health, an effect far in excess of preventive measures that affect only individual forms of late-life disease such as cancer or heart disease (Olshansky et al., 1990, 2006; Miller, 2002). The published literature contains sporadic reports (Schneider \& Miller, 1998; Schneider \& Reed, Jr, 1985 ) of dietary additives purported to have beneficial effects in rodent models, but none that has been replicated and accepted by the scientific community as a reliable and reproducible result. Stimulated by a consensus conference in 1999 (Warner et al., 2000), the National Institute on Aging (NIA) has developed a program, the NIA Interventions Testing Program (ITP), to evaluate agents that are considered plausible candidates for delaying aging or preventing multiple forms of late-life disease in laboratory mice. This article presents the central features of the program, now in its fourth year of operation, along with an interim analysis of survival data for mice exposed to four agents: aspirin, nitroflurbiprofen (NFP), 4-OH- $\alpha$-phenyl-N-tert-butyl nitrone (4-OH-PBN), and nordihydroguiaretic acid (NDGA).

We describe here central features of the ITP protocol: selection of test agents, simultaneous replication at three test sites, use of genetically heterogeneous mice, use of both pair-fed and 
untreated controls, consideration of statistical power, inclusion of age-sensitive traits, plans for necropsy analysis, and plans for interim analyses of survival.

\section{Selection of test agents}

The NIA makes an annual announcement requesting suggestions of interventions that may be suitable for inclusion in the ITP protocol. The most recent announcement in the $\mathrm{NIH}$ guide can be found at grants. nih.gov/grants/guide/notice-files/NOT-AG-07003.html, and additional information about the selection process can be found at www.nia.nih.gov/Researchlnformation/ ScientificResources/InterventionsTestingProgram.htm.

Individual scientists, including those affiliated with governmental, academic, or commercial research groups, are asked to nominate compounds they think would be worth evaluating in the ITP, to specify the grounds for their recommendation, and to provide advice on the best dosage regimen for the study. These suggestions are considered by an ITP access committee, whose recommendations are then transmitted to the program's steering committee for final approval. The basis for nomination of a test agent may include a mixture of theoretical ideas and empirical data. Some compounds are tested because they have shown promising results in small scale studies of rodents, or comprehensive studies in worms or flies; others are recommended because they affect biochemical or physiological processes, like inflammation or oxidation or stress resistance or insulin sensitivity, that are thought to be involved in regulation of aging. Practical considerations prompt greater enthusiasm for interventions that can be given in food or water and are relatively inexpensive. Interventions that involve more intensive effort, such as injection or implantation procedures or environmental manipulations, can be considered if their biological effect is thought likely to require only transient or periodic exposure rather than daily intervention.

\section{Parallel studies at three institutions}

The design of the program emphasizes the value of simultaneous replication at multiple test sites. Investigators interested in antiaging interventions, either as probes of the aging process or as a step towards developing preventive medicines for human use, are likely to have greater confidence in a report that includes consistent data from multiple sites than they would in the typical report that includes data from a single research site only. For this reason, each agent accepted for the ITP is evaluated at three sites: the Jackson Laboratory (TJL), the University of Michigan at Ann Arbor (UM), and the University of Texas Health Science Center at San Antonio (UT). The scientific staff at each of these three sites have attempted to replicate as carefully as possible the protocol in use at the other two sites, for example, by ordering bedding from a single supplier, preparing control and experimental diets from the same supplier and shipped from a single source, co-ordinating environmental variables such as light/dark cycles and temperature, etc.

\section{Use of genetically heterogeneous mice}

ITP uses mice produced by a four-way crossbreeding scheme, in which the experimental animals are the progeny of CB6F1 females bred to C3D2F1 males. Each animal in the test population is thus genetically unique, but each shares half of its nonmitochondrial genome with every other test mouse; from this perspective, all the animals are full sibs. This specific four-way cross (UM-HET3) has been used previously for a series of biomarker and gene mapping analyses (Jackson et al., 1999; Miller et al., 1999a; Harper et al., 2003; Hanlon et al., 2006), providing some background information against which ITP data sets can be compared. In addition, a set of single nucleotide polymorphism genotyping probes should allow relatively inexpensive quantitative trait locus mapping studies of any trait measured in the ITP mice. The rationale for using genetically heterogeneous mice instead of one of the commonly used inbred mouse strains is to avoid missing true positive effects of agents that might fail to work in a specific inbred genotype, and to reduce the chance of giving undue emphasis to an agent that might work only in the specific inbred stock selected (Committee on Animal Models for Research on Aging, 1981; Miller et al., 1999a,b). Male and female mice are tested in parallel at each institution.

\section{Use of pair-fed and untreated controls}

Each year's work includes animals exposed to one of a series of interventions (typically 4, 5, or 6 per year), plus a group of untreated controls, plus a group of mice whose food supply has been reduced to a level that allows these 'pair-fed' mice to track the weight trajectory of mice with the lowest mean weight of the experimental groups. The number of untreated control animals is approximately twice the number of those in any one of the treatment groups, to provide increased statistical power for tests of treated against control mice for life table analyses; use of an unbalanced design of this kind is a cost-effective way to increase statistical power in that the same oversampled control group is used for comparisons against each of the experimental groups. The pair-fed group is intended to provide a basis for comparison for any agent that leads to diminished body weight. This comparison may, in some cases, permit rejection of the idea that an agent increases lifespan merely by reduction of appetite and thus by inducing a form of caloric restriction, by allowing a comparison of life table to mice with a similar weight trajectory but which have not been exposed to the intervention in question. None of the agents tested in the first year's group was found to lead to diminished mean weights, and mice in the pair-fed group were therefore removed from the protocol and used for other purposes. Thus none of the figures or tables in this report include any information about survival of mice originally assigned to the pair-fed group.

\section{Consideration of statistical power}

The ITP standard protocol enrolls 36 female and 44 male mice in each treatment group, at each of the three test sites, an equal 
number in the pair-fed populations, and includes 72 females and 96 males in the untreated control group. Oversampling of males is intended to compensate for the larger number of earlylife deaths of male mice due to fighting; the protocol dictates euthanasia for all mice in a cage containing an animal severely injured by fighting. This protocol was the result of a formal power analysis, considering a variety of analytical end-points and alternative designs, conducted by Drs Andrzej Galecki (University of Michigan) and Scott Pletcher (Baylor College of Medicine). According to the power calculations, a design of this size is sufficient to detect a $10 \%$ increase (or decrease) in mean lifespan with $80 \%$ power with respect to unmanipulated controls of the same sex, even if data from one of the three test sites are unavailable (e.g. because of a catastrophic failure of temperature or infection control).

\section{Evaluation of age-sensitive traits}

The consensus conference from which the ITP program developed (Warner et al., 2000) included much discussion of what proportion of resources to commit to testing age-sensitive traits in addition to lifespan. Some researchers advocated evaluation of a wide range of physiological and biochemical end-points in each test group, in the hopes of detecting beneficial effects of agents independent of any effect on longevity, while others thought that doing so would, because of budgetary constraints, limit the number of agents (and doses) that could be tested in any one year. The principal goal of the ITP is to find interventions that slow aging using extension of maximum lifespan as a surrogate measure for aging rate. For this reason the ITP group has decided to include only a small number of mid-life end-points in its initial testing, reserving more detailed characterization of multiple systems for later studies of interventions that appear promising in the initial screens. Even the initial populations ('Phase I' tests), however, are evaluated for three age-sensitive traits. (i) A sample of mice (50 per test group +100 controls) is tested at ages 7 and 18 months for spontaneous locomotor activity, during a 50-h period in a standard mouse cage, using a protocol that permits independent assessment of ambulation, stereotypic movements (in place), and day/night distribution of activity. (ii) A blood sample taken at 18 months is evaluated for proportions of four age-sensitive T-lymphocyte subtypes. (iii) Serum taken at 18 months is evaluated for a series of hormones including insulin, insulin-like growth factor I (IGF-I), leptin, and thyroxine $\left(\mathrm{T}_{4}\right)$.

\section{Plans for necropsy analysis}

Details of pathological lesions are likely to be of use for evaluation of any agent that extends lifespan in the ITP protocol, and potentially for agents that unexpectedly lead to shorter lifespan than that seen in the control groups, but unfortunately it will not be possible to learn which agents have these effects until most of the mice have died. For this reason, the ITP protocol involves fixation of the bodies of mice found dead (or euthanized when severely moribund), and their preservation for future study if necessary.

\section{Plans for interim analyses}

We plan to conduct our primary lifespan analysis when we have a sufficient number of deaths to let us test hypotheses about maximum lifespan. Analytical plans that include frequent evaluation of the same evolving data set at multiple intervals have well-known disadvantages, in particular the inflation of overall Type I error rate. An interim analysis, however, presents important advantages, in that it may alert the scientific community to agents that postpone early-life deaths and may deserve increased scrutiny for possible effects on aging, age-sensitive traits, and physiological end-points thought to be important in the aging process. We have therefore elected to conduct an interim analysis of survival data at a preselected time-point, specifically using a data set frozen on the day that $50 \%$ of the control mice have died at the site where this criterion is last met. Separate interim analyses will be conducted for male and for female mice, using the survival curve of the corresponding control population in each case. This report, for example, is based upon the survival data available on December 30, 2006, the date on which at least $50 \%$ of the male control mice were dead at each of the three test sites.

\section{Test agents for Cohort 1}

For the initial cohort, four agents were selected: aspirin, NFP, NDGA, and 4-OH-PBN.

Chronic inflammation is associated with many chronic diseases of aging, and may actually contribute to those diseases. Changes with age in both adaptive and nonadaptive immunity may alter both structures and functions of organs and tissues, while chronic diseases of aging may stimulate inflammation in a deleterious feedback system. Inflammatory cytokines and other mediators of inflammation can also be strong near-term predictors of mortality associated with age-related chronic diseases. An inflammatory component is involved in the pathogenesis of many age-associated diseases such as atherosclerosis (Weissberg \& Bennett, 1999), Alzheimer's disease (Akiyama et al., 2000), many forms of cancer (Masferrer et al., 2000), and osteoarthritis (Pincus, 2001). Aging also is characterized by an elevation in such proinflammatory cytokines as interleukin 6 (IL-6) and tumor necrosis factor- $\alpha$ (TNF- $\alpha$ ) (Spaulding et al., 1997; Ershler \& Keller, 2000), and high levels of IL-6 are powerful predictors of morbidity and mortality in the elderly (Ferrucci et al., 1999; Harris et al., 1999). In addition, caloric restriction, the best-characterized antiaging intervention, consistently reduces inflammatory responses. Two anti-inflammatory agents were included in the first set of ITP test compounds: aspirin and NFP.

Aspirin, a nonsteroidal anti-inflammatory drug (NSAID), also has antithrombotic and antioxidant properties (Weissmann, 1991; Shi et al., 1999; Vane, 2000). Some of its effects represent inhibition of cyclooxygenase I (COX-I) and cyclooxygenase ॥ 
(COX-II), which synthesize prostaglandins through enzymatic oxidation of arachidonic acid. At therapeutic doses, aspirin suppresses production of TNF- $\alpha$ by an effect on NF- $\mathrm{KB}$ signals (Shackelford et al., 1997). Aspirin may also have indirect effects on oxidative damage to proteins by acetylation of $\varepsilon$-amino residues, thus blocking inter- and intra-protein crosslinks as well as glycation reactions (Jones \& Hothersall, 1993; Weber et al., 1995; Shi et al., 1999; Caballero et al., 2000). Aspirin at $100 \mathrm{mg} \mathrm{kg}^{-1}$ per day was found to reduce carbonyl formation and bityrosine formation in the lens crystallins of diabetic rats, presumably by an antioxidant effect (Jones \& Hothersall, 1993); formation of advanced glycation end-products was similarly inhibited. Aspirin at concentrations of $333 \mathrm{mg} \mathrm{kg}^{-1}$ body weight per day prevented the development of both cirrhosis and preneoplastic and neoplastic nodules, but without any directly associated prevention of fatty changes in male F344 rats (Denda et al., 1994).

Nitroflurbiprofen is a nitric oxide (NO)-releasing flurbiprofen derivative (Ongini \& Bolla, 2006). Like the commonly used NSAIDs such as ibuprofen, NFP has about equal inhibitory potential against COX-I and COX-II. It crosses the blood-brain barrier, so may benefit chronic inflammation in the central nervous system (CNS). The NO-releasing moiety of NFP confers protection against gastrointestinal toxicity, protects the cardiovascular system, and enhances the anti-inflammatory effect, therefore making the drug safer than reference NSAIDs in conditions requiring chronic administration. Although longterm anti-inflammatory treatments can sometimes damage kidney and heart tissues, previous studies have shown that 6-12 months of exposure of mice to NFP did not produce overt signs of toxicity (van Groen \& Kadish, 2005; Brunelli et al., 2007). The dose of $30 \mathrm{mg} \mathrm{kg}^{-1}$ per day used in previous studies was fully effective in a variety of experimental paradigms involving activity in both NO and COX pathways; these data provided a basis for the dose used in the ITP testing program. Early human studies have also suggested that NFP may have a favorable safety profile for the gastrointestinal and cardiovascular system (Ongini \& Bolla, 2006).

Nordihydroguaiaretic acid (NDGA; also called masoprocol) is a naturally occurring dicatechol produced by the creosote bush Larea tridentata. NDGA has both antioxidant and antiinflammatory properties, and resembles other polyphenols, including resveratrol, that can activate sirtuins and have shown life-extension properties in metazoan models (Wood et al., 2004). NDGA inhibits arachidonic acid 5-lipoxygenase and its cytokine-stimulated activation of microglia or macrophages, in part by suppression of proinflammatory gene expression and prostaglandin $\mathrm{E}_{2}\left(\mathrm{PGE}_{2}\right)$ production (West et al., 2004). NDGA therefore might inhibit age-related tissue damage arising from inflammatory products of arachidonic acid metabolism. One group has reported that low concentrations of NDGA can extend mean and median adult lifespan of mosquitoes by $50 \%$ (Richie, Jr, et al., 1986). Similarly, NDGA reportedly extends lifespan by $10 \%$ in Drosophila (Miquel et al. 1982) and extends clonal lifespan in Neurospora crassa (Munkres \& Colvin, 1976). Only one report exists of NDGA effects on age-related mortality in a mammal, a study in which Wistar rats received food containing 0.005\% NDGA (Buu-Hoi \& Ratsimamanga, 1959); after 2 years of treatment, eight of 12 remained alive in the NDGA group, compared to two of 12 control animals. In a separate study, food containing $0.1 \%$ NDGA was reported to slow disease progression and thus increase lifespan in mice with motor neuron disease caused by a mutation in superoxide dismutase 1 (West et al., 2004). Nontransgenic mice fed the same NDGAsupplemented diet were found to tolerate the drug chronically and demonstrated a tendency for enhanced motor function at later ages ( $K$. Hensley, personal communication). These observations, while fragmentary, provided a rationale for evaluating the potential effect of NDGA on lifespan and health in the ITP.

Nitrones, with the general formula $\mathrm{X}-\mathrm{CH}=\mathrm{NO}-\mathrm{Y}$, have been used for over 30 years in analytical chemistry to trap and stabilize free radicals. One such agent, PBN ( $\alpha$-phenyl-N-tertbutyl nitrone), has been shown to have potent pharmacologic activities in experimental models of age-related diseases including stroke (Floyd, 1990; Zhao et al., 1994) and cancer (Floyd et al., 2002; Nakae et al., 2003). The principal metabolite of PBN is its 4-hydroxy derivative, 4-OH-PBN, which was evaluated in the ITP. In one study (Edamatsu et al., 1995), PBN at $30 \mathrm{mg} \mathrm{kg}^{-1}$ per day was found to extend lifespan of the short-lived stock SAM-P8 by about 33\%. A second study (Saito et al., 1998) documented a beneficial effect of PBN at about $38 \mathrm{mg} \mathrm{kg}^{-1}$ per day on longevity in C57BL/6J mice when treatment was initiated at 24.5 months of age. In a third study (Sack et al., 1996), PBN was administered at $32 \mathrm{mg} \mathrm{kg}^{-1}$ body weight per day to SpragueDawley rats, starting at age 24 months, and was shown both to improve memory retention and to increase median lifespan from 29.4 to 32.2 months.

We report here the results of a planned interim analysis of survival, conducted on the data set as it stood on December 30,2006 , the date at which at least $50 \%$ of the male control mice had died at each of the three test sites.

\section{Experimental procedures}

\section{Mice}

Mouse production, maintenance, and estimation of lifespan UM-HET3 mice were produced at each of the three test sites. The mothers of the test mice were CByB6F1/J, JAX stock $\# 100009$, whose female parents are BALB/CByJ and whose male parents are $\mathrm{C} 57 \mathrm{BL} / 6 \mathrm{~J}$. The fathers of the test mice were C3D2F1/J, JAX stock \#100004, whose mothers are $\mathrm{C} 3 \mathrm{H} / \mathrm{HeJ}$ and whose fathers are DBA/2J. The first litter from each breeding cage was discarded, so that all of the experimental animals would be the product of a second or subsequent pregnancy. This decision was made to avoid the possibility that mice that were born to primiparous dams might receive inferior nutrition or maternal care compared to products of subsequent pregnancies. Each test site entered approximately equal numbers of 
weanlings each month over a 6-month period. Mice were weaned into same-sex cages (three males or four females per cage) at the age of 19-21 days. Sires were not separated from pregnant dams during the period of pregnancy, to allow subsequent impregnation at postpartum estrous. Mice did not receive ear punches or toe clips or other identifying marks at weaning. Each site used diets based on the NIH-31 standard. For breeding cages, UM used Purina 5008, UT used Teklad 7912, and TJL used Purina 5K52. For weanlings prior to 4 months of age, UM used Purina 5001, UT used Teklad 7912, and TJL used Purina 5LG6.

Mice were housed in plastic cages with metal tops, using corn-cob bedding, specifically 1/4 inch Bed O'Cobs, produced by The Andersons, PO Box 114, Maumee, Ohio. Mice were given free access to water, acidified $(\mathrm{pH} 2.5-2.7)$ by addition of hydrochloric acid, using water bottles rather than an automated watering system. Mice were housed in ventilated cages, and were transferred to fresh cages every 14 days, except that at UT mice were transferred to fresh cages every 7 days. Temperature was maintained within the range of $21-23^{\circ} \mathrm{C}$.

At the age of 42 days, each cage was assigned to a control or test group by use of a random number table. Each mouse was then briefly anesthetized by isoflurane inhalation administered either by nose cone or by an instrument designed for small animal anesthesia. Measures were taken of weight, body length, and tail length, and an electronic ID chip was implanted by sterile syringe beneath the dorsal skin between the shoulder blades, after which the wound was closed by a drop of superglue (Loctite gel, purchased locally, or Nexaband S/C, purchased from Abbott Laboratories, North Chicago, IL, USA). UM and UT used chips purchased from AVID Microchip ID Systems (Folsom, LA, USA; catalog AVID3002); TJL used chips purchased from Locus Technology (Manchester, MD, USA; catalog 1D-100A). A portion of the distal tail $(1 \mathrm{~cm})$ was taken and frozen for later analysis of DNA polymorphisms, after which the mouse was permitted to awaken from the anesthesia. The duration of anesthesia was approximately 1-2 min.

Mice received control diet until the age of 4 months, after which experimental diets were substituted for mice in the NFP, 4-OH-PBN, and aspirin groups. Mice in the NDGA group started receiving NDGA at the age of 9 months.

At the age of 7 months, a subset of the mice (100 controls plus 50 of each test group at each site) were evaluated for activity, using a test in which each mouse is housed individually in a standard mouse cage for $50 \mathrm{~h}$, while a computer records episodes of ambulation or movement in place. This test was repeated, at the age of 18 months, for each live mouse that had been previously tested. Three weeks after the activity test, a blood sample was obtained from each mouse that had been tested for activity; these samples were used for analyses of $T$ cell subsets and hormone levels that will be reported elsewhere.

Each colony was evaluated for the presence of pathogens four times each year. The test procedure involves pooling used bedding from groups of cages, and exposing sentinel mice to the spent bedding for a period of 6 weeks. Blood samples from the sentinel mice are then examined for antibodies to mouse viruses, and the sentinels themselves tested for pinworm eggs. At UM, the mice are evaluated quarterly for antibodies to mouse hepatitis virus (MHV), minute virus of mouse (MVM), mouse parvovirus (MPV), rotavirus (EDIM), and ectromelia, and pinworms are sought in a cecal exam. In addition, tests are done semiannually for Sendai, pneumonia virus of mice (PVM), Theiler's virus (TMEV, GD VII), reovirus 3 (REO), Mycoplasma pulmonis (MPUL), lymphocytic choriomeningitis virus (LCMV), mouse adenovirus (MAV), and polyoma virus. TJL tests for all of these agents quarterly, plus K virus, Hantaan virus, lactic dehydrogenase elevating virus, mouse cytomegalovirus (MCMV), and mouse thymic virus (MTV). In addition, TJL evaluates serum for ciliaassociated respiratory (CAR) bacillus, evaluates tracheal cultures for Streptobacillus moniliformis, and evaluates fecal or intestinal cultures for Citrobacter rodentium and Salmonella spp. The testing protocol at UT is similar, but also includes tests for several species of Helicobacter. All such surveillance tests were negative at all three sites throughout the experiment.

\section{Removal of mice from the longevity population}

Mice were inspected daily, and husbandry staff made a notation when they found an animal with severe bite wounds, that is, wounds that were present on more than $20 \%$ of the skin or that were bleeding or infected. If the staff noted continued evidence of wounding over the next 2-week period, all mice in the cage were euthanized. Thirty-six mice were removed from the study for this reason, including 33 males and three females; of these 36 mice, three were at TJL ( $0.5 \%$ of 654 TJL mice), 20 ( $3 \%$ of 658 ) at UM, and 13 ( $2 \%$ of 657 ) at UT. An additional 26 mice were removed because of an accident, typically either death during chip implantation or failure of the chip to function (nine at TJL, eight at UM, nine at UT). An additional 51 mice were removed from the longevity protocol when they were used for another experimental purpose, such as testing for blood levels of a test agent (33 at UM and 18 at UT). For survival analyses, all such mice were treated as alive at the date of their removal from the protocol, and lost to follow-up thereafter. These mice were not included in calculations of median longevity.

\section{Estimation of age at death (lifespan)}

Mice were examined at least daily for signs of ill health. Mice were euthanized for humane reasons if they are so severely moribund that they are considered, by an experienced technician, unlikely to survive for more than an additional $48 \mathrm{~h}$. A mouse was considered severely moribund if it exhibited more than one of the following clinical signs: (i) inability to eat or to drink; (ii) severe lethargy, as indicated by reluctance to move when gently prodded with a forceps; (iii) severe balance or gait disturbance; (iv) rapid weight loss over a period of 1 week or more; or (v) an ulcerated or bleeding tumor. The age at which a moribund mouse was euthanized was taken as the best available estimate of its natural lifespan. Mice found dead were also noted at each daily inspection. 

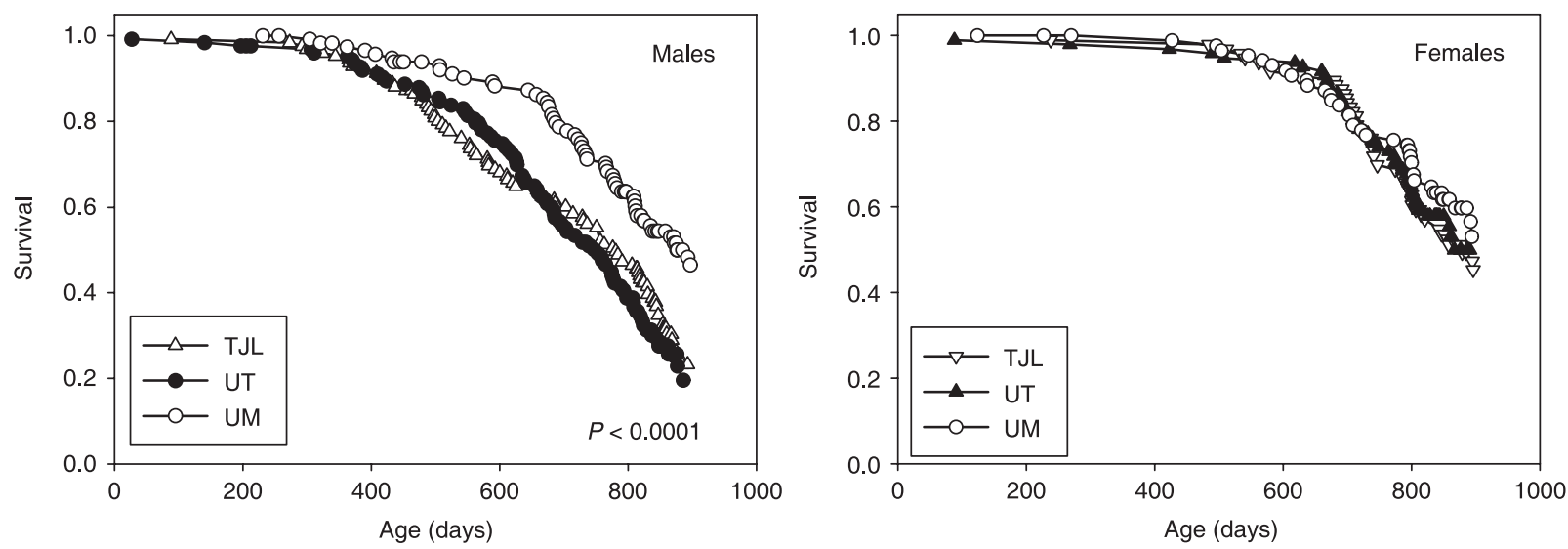

Fig. 1 Survival curves for male and female mice, in the control group, at each test site. Data are shown only for ages up to 900 days. For males, log-rank $P<0.0001$ for the hypothesis that all three sites show the same survival distribution.

\section{Control and experimental diets}

The test substances were incorporated into Purina 5LG2 chow, an irradiated diet containing $4 \%$ fat, based on the $\mathrm{NIH}-31$ standard. Experimental diets were administered to the mice from 4 months of age (except for NDGA, which was initiated at 9 months of age). Purina prepared batches of food containing each of the test substances, as well as control diet batches, at intervals of approximately 4 months, and shipped each batch of food at the same time to each of the three test sites. NDGA was purchased from Cayman Chemicals (Ann Arbor, MI, USA) and mixed with chow at a concentration of 2500 milligrams of NDGA per kilogram of food. Nitroflurbiprofen was obtained from NicOx Research Institute (Milan, Italy) and used at a dose of 200 milligrams NFP per kilogram of food. Aspirin was obtained from a local supplier, and used at a dose of $21 \mathrm{mg}$ per $\mathrm{kg}$ of food. 4-OH-PBN was synthesized in the laboratory of Robert Floyd, and used at a dose of $350 \mathrm{mg}$ per $\mathrm{kg}$ of food. On the assumption that each mouse weighs $30 \mathrm{~g}$ and consumes $5 \mathrm{~g}$ food per day, the estimated daily doses of these agents would be NDGA 417; NFP 33; 4-OH-PBN 53, and aspirin $3.3 \mathrm{mg} \mathrm{kg}^{-1}$ body weight per day.

\section{Statistical methods}

Each mouse originally entered into the study was, at the time of analysis, considered to be in one of three categories: alive, dead (from natural causes), or culled. Mice were considered to be culled at the age at which they were no longer subjected to the mortality risks typical of unmanipulated mice. In some cases this was because the mouse was removed because of fighting; in other cases mice died as the result of an accident (e.g., death when anesthetized for implantation of a radio-emitting chip). In further cases mice were considered 'culled' on the day in which they received an experimental treatment (such as blood sampling or injection with an inflammatory agent) to which the control mice were not exposed. Kaplan-Meier analysis, and log-rank comparisons among groups consider 'culled mice' to be lost from follow-up on the day at which they were removed from the longevity protocol, and consider mice alive on the day of analysis (December 30, 2006) as lost to follow-up at that time.

Unless stated otherwise, all significance tests about survival patterns are based upon the two-tailed log-rank test at $P<0.05$, with culled mice included up until their date of removal from the longevity population.

Other statistical tests are described in the text; all $P$ values were two tailed.

\section{Results}

Of the 1100 male mice entered into the study (distributed in roughly equal numbers among the three test sites), 1029 remained unculled at the interim analysis point; of these 1029 mice, 650 (63\%) were dead. Of the 869 females originally enrolled, 827 remained unculled at the analysis point; of these 827 animals, 462 (56\%) were dead. The oldest surviving mice at the analysis point were 32.5 months of age, and the youngest were 26 months.

Figure 1 shows the survival curves for control males and females at each of the three test sites. Survival for females is higher than for males at TJL and at UT $(P<0.0001$ at each site), but not at UM $(P=0.32)$. There is no significant difference among the three sites in survival for control females, but control males at UM were significantly more likely to survive than control males at the other two sites $(P<0.0001)$. There were no significant differences between UT and TJL in survival curves for either sex.

Table 1 shows median survival times for male and female control mice at each site, with culled mice excluded from the calculation. We do not at present understand the basis for the relatively high mortality risks at TJL and UT compared to UM, nor why these risks affect male mice but not females.

Figure 2 shows survival curves for male mice, pooling data across all three sites and including culled animals, for controls as compared to mice in each of the four experimental groups. A log-rank test, comparing among all five groups, showed that the differences among groups were significant at $P<0.005$, 
Table 1 Median survival values for male and female control mice

\begin{tabular}{lll}
\hline Site & Sex & Median \pm standard error $(n)$ \\
\hline TJL & F & $858 \pm 7(93)$ \\
UM & F & $909 \pm 4(86)$ \\
UT & F & $876 \pm 4(96)$ \\
TJL & M & $781 \pm 7(125)$ \\
UM & M & $876 \pm 8(106)$ \\
UT & M & $739 \pm 7(119)$ \\
\hline
\end{tabular}

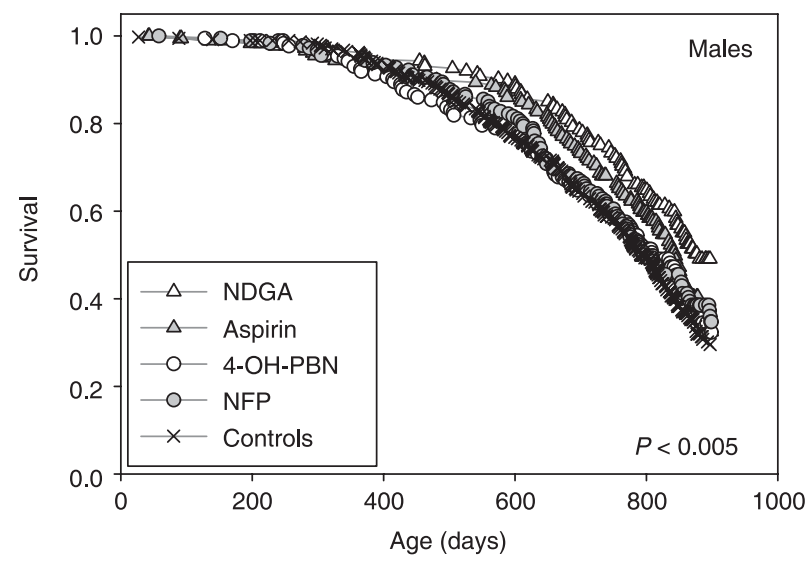

Fig. 2 Survival curves for male mice, pooled across all three test sites, by each of the four tested interventions, plus controls. Data shown up to the age of 900 days. Log-rank $P<0.005$ for the hypothesis that all five treatment groups show the same survival distribution.

using a calculation stratified by test site. A secondary analysis then repeated this comparison separately for the male mice at each test site, and showed a significant difference among experimental groups for male mice at TJL $(P<0.01)$ and UT $(P<0.04)$, but not at UM.

We then repeated the stratified log-rank test, for male mice, pooling across sites, to compare the control group with each of the individual test agents. For NDGA, $P=0.0004$ for the pooled data. The NDGA effect was significant for TIL and UT males at $P=0.02$ and $P=0.005$, respectively, but was not significant for males at UM. Figure 3 shows comparisons of NDGA to control mice for males at each of the three test sites.

There were no significant effects of $4-\mathrm{OH}-\mathrm{PBN}$ or of NFP in male mice, pooling across sites. For aspirin, the log-rank test showed a trend toward improved survival $(P=0.07$, pooled data), with site-specific $P$ values for TJL, UM, and UT of $P=0.05$, 0.64 and 0.17 , respectively. We conducted a secondary analysis using the Wilcoxon-Breslow test (which gives greater weight to deaths at early time-points) instead of the log-rank test, and found $P=0.03$ for the pooled data set.

Figure 4 shows a comparison among test groups for female mice, pooling across test sites. The log-rank test did not show any evidence for significant differences attributable to treatment group for the female mice.

It is possible that some dietary interventions might cause a decline in food palatability, leading to a decline in food
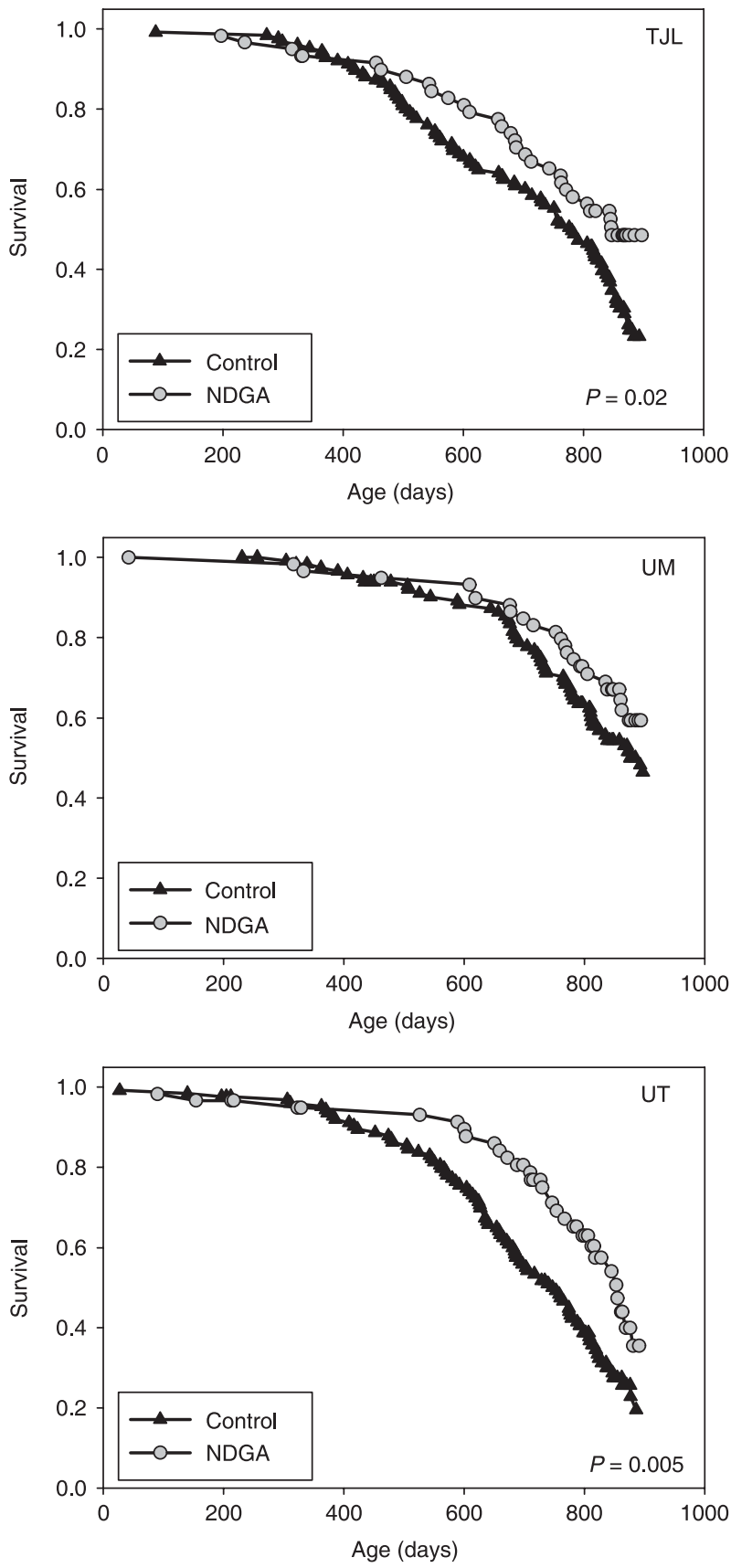

Fig. 3 Survival curves comparing nordihydroguiaretic acid (NDGA) treatment to control mice, for males only, for each of the three test sites. Data shown up to the age of 900 days. Log-rank $P$ values refer to the comparison of NDGA males to control males.

consumption and thus to a form of voluntary caloric restriction. Although neither food consumption nor adiposity was measured in this pilot study, we did measure weight in each mouse at ages $6,12,18$, and 24 months, and at some sites also at ages $1.4,3$, and 4 months, as an indirect measure of nutritional status. The data are presented separately for each site, and for both males and females, in Fig. 5. Considering the control groups, both male and female mice at UM were significantly lighter in 


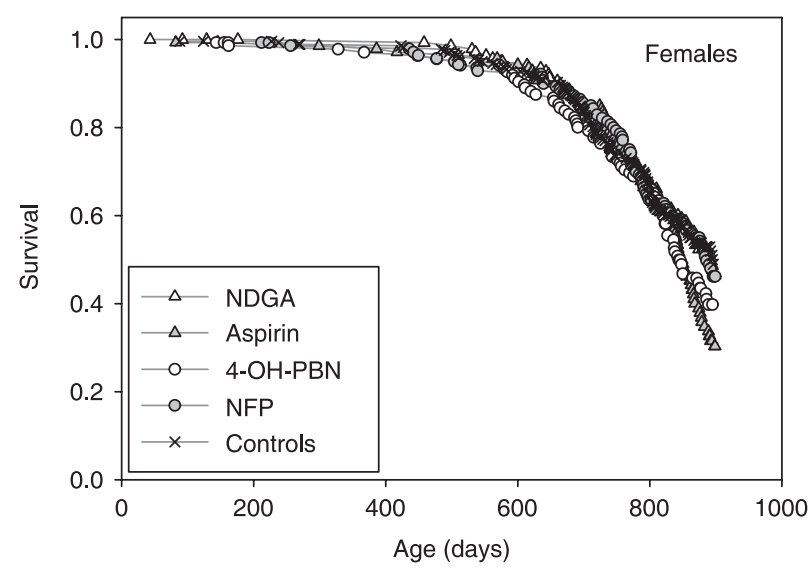

Fig. 4 Survival curves for female mice, pooled across all three test sites, by each of the four tested interventions, plus controls. Data shown up to the age of 900 days.

weight than mice at either of the other two test sites at each age from 6 months onward, even though each site used the same batches of food prepared and shipped from the same diet kitchen. The difference between mice in the NDGA and control groups at UM, seen at 6 months, cannot be attributed to NDGA effects, because the mice assigned to the NDGA group were not actually exposed to this agent prior to 9 months of age. At 12 months of age there was a small, but statistically significant difference between control and NDGA mice at the UM site $(1.9 \mathrm{~g}, P<0.02)$, but this is smaller than the difference seen at this site $(4.4 \mathrm{~g})$ at 6 months of age, that is, prior to NDGA administration, and so is unlikely to represent a drug effect per se. There were no significant effects of NDGA at UT or TJL, in male or female mice. These data do not support the idea that the beneficial effects of NDGA on survival are mediated by caloric restriction.

\section{Discussion}

Our results suggest that NGDA diminishes mortality risks for male UM-HET3 mice over the first half to two-thirds of the expected lifespan. The primary analysis, focused on the pooled data set, showed that this effect was highly significant, and confidence in the result was bolstered by noting that the effect was statistically significant at two of the test sites, and showed a similar (though nonsignificant) trend at the third test site. It is noteworthy, however, that the two sites with the strongest effects of NDGA were those at which the control male mice had the highest risk of mortality. The effect of NDGA, at this interim time-point, also seems to be limited to male mice, contrary to our working hypothesis that any beneficial effects of test agents would be seen in mice of both sexes. Based on the body weight data, it seems very unlikely that the effect of NDGA is mediated by caloric restriction. None of the other three tested agents had a significant effect on survival at this interim test point, although there is a suggestion that aspirin might have a small benefit for early survival in males. Because of cost
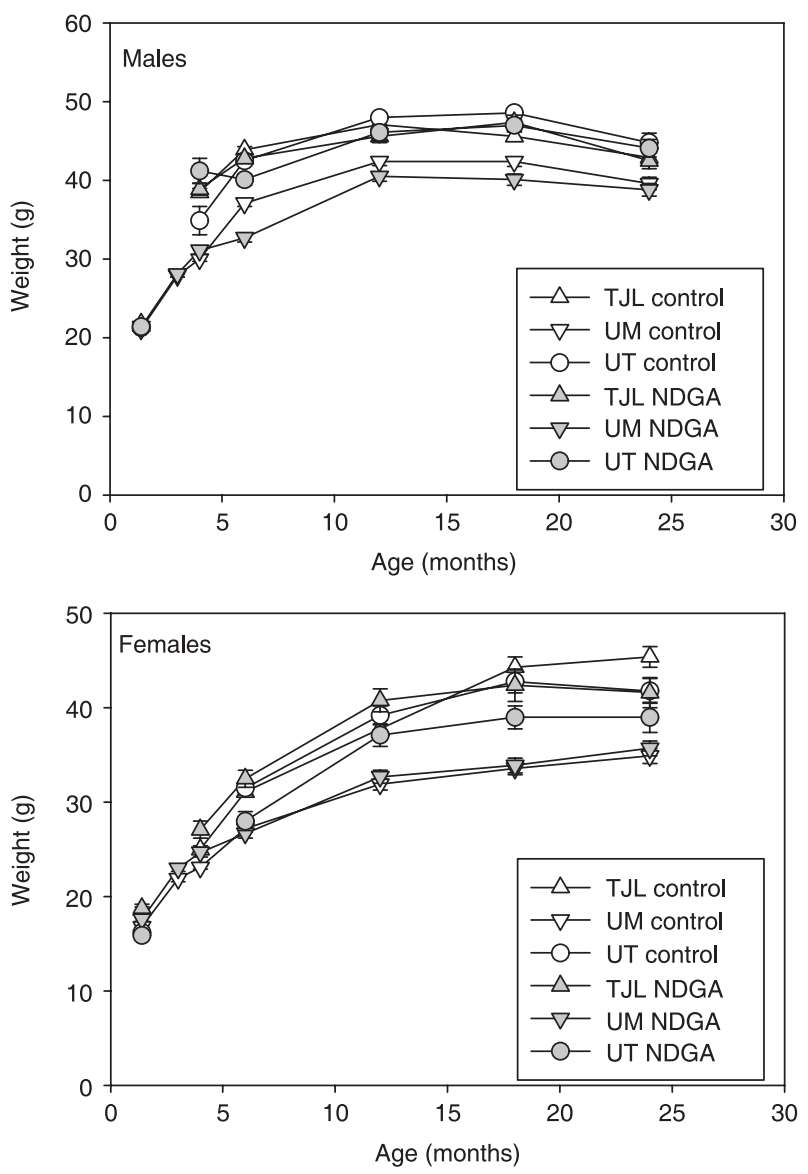

Fig. 5 Mean weight as a function of age, comparing nordihydroguiaretic acid (NDGA) to control mice, at each of the three test sites. Top panel shows males, and bottom panel shows females. Each symbol shows mean \pm SEM for 2460 mice in each NDGA group, and for 46-125 mice in each control group (depending on age and site).

considerations, we were only able to test each compound at a single dose, and the dose selected was in each case based on only a limited amount of previous experience with the agent or its congeners in rodents. It is possible that different results, potentially including a positive effect on mid-life survival risks, might have been seen at doses higher or lower than those used in this study.

Although there have been many prior reports in which agents added to rodent chow have been seen to extend median or maximum lifespan (see Schneider \& Miller, 1998; Schneider \& Reed, 1985 for reviews), so far as we know none of these have been replicated, either at the original test site or at a different site. The design of the ITP features simultaneous replication at three independent sites, and it is noteworthy that the NDGA effect indeed does seem to be similar at all three sites, with a statistically significant effect at the two sites with the largest number of recorded deaths at this interim time point.

We wish to emphasize that these results do not address the central goal of the ITP, which is to determine if any of the test agents slow aging to an extent sufficient to increase maximum lifespan. There are circumstances in which an intervention, such 
as exercise (Holloszy, 1993), has been shown to increase median lifespan or some other index of early-life mortality risks, without any effect on maximum lifespan or an equivalent index of survival among the oldest test subjects. NDGA might, for example, act by postponing one or more specific forms of lethal early-life illness. We anticipate that $90 \%$ of the control population is likely to have died by about 32 months of age, and that the youngest group of mice in our study will have reached this age by July 2007. At this point, it will be possible to assess the effect of treatments on the proportion of mice that live past the age at which $90 \%$ of the population has died; a significant effect using a quantile test (Wang et al., 2004) might then support the idea that one or more of the agents may retard aging. No such conclusion would, however, be warranted at this stage.

We do not understand why median survival for male mice is higher at UM than at UT or TJL, nor why the factors that contribute to this difference among sites affect males but not females. Historical control data are available from three prior longevity studies performed on male and female virgin UMHET3 mice at the University of Michigan, from which median survival values can be calculated. One such study (Jackson et al., 2002) found median survival of 787 days for males and 818 days for females, excluding the male mice that died at early ages from urinary syndrome. A second study (Miller \& Chrisp, 1999) had median survival of 829 days for males and 836 days for females. The third, and largest, population (Harper et al., 2004) had median levels of 862 days for males and 864 days for females. Comparison of these historical values to the data in the present study is not straightforward, because of differences in protocols, including, for example, the elimination of first litters from the ITP study. The median value for ITP control males at UM (876 days) is similar to that seen in the most recent of the prior populations, and the median levels for control males at UT and TJL (739 and 781 days) are somewhat lower than those seen in any of the three UM-HET3 lifespan experiments, all of which were conducted at UM. In contrast, the values for female control mice (858, 876, 909 for the three ITP sites) are similar to or slightly higher than those seen in the previous UM-HET3 populations, suggesting that the variation among the male populations is unlikely to represent any systematic problems with husbandry operations or standards of health care in any of the colonies. Each of the three test sites used a different variation of the $\mathrm{NIH}$ 31 diet for breeding cages, and for weanling mice prior to the initial exposure to experimental and control diets, and it is possible that variations in vitamin levels, salts, and protein sources at these early ages might have contributed to differences among sites in male survival and male and female weight trajectories. There are a small number of other known inconsistencies among the sites, such as the use of different brands of implanted microchips, and the schedule of cage changes ( 14 days at TJL and UM, 7 days at UT), and there are likely to be changes in other environmental variables (noise levels, light or temperature gradients, odors) that are not apparent to investigators but could potentially affect health of the mice. The dramatic variation among sites in body weights of both male and female animals, despite the use of rodent chow shipped to each site, in parallel, from a single source, testifies to the presence of uncontrolled environmental factors with potent effects on key metabolic endpoints, and thus to the importance of replication of life table data at independent test facilities.

NDGA is a product of the creosote bush, L. tridentata, which grows in the southwestern USA and northern Mexico. It has a wide variety of uses in folk medicine including treatment of infertility, rheumatism, arthritis, diabetes, gallbladder and kidney stones, pain, and inflammation. It is a potent antioxidant and was widely used in the 1950s as a food additive to prevent spoilage, until it was banned because of reports of toxicity in the 1960s (Arteaga et al., 2005). It has been reported to increase lifespan in mosquitoes, fruit flies, and rats and to prolong the survival of the G93A mouse model of amyotrophic lateral sclerosis (Buu-Hoi \& Ratsimamang, 1959; Miquel et al., 1982; Richie et al., 1986; West et al., 2004). In addition to its antioxidant properties, there are several actions of NDGA that could lead to beneficial effects. NDGA has a spectrum of activities similar to that of other naturally occurring biphenolic compounds such as resveratrol. It is widely reported to be a potent anti-inflammatory agent through its inhibitory action on 5-lipoxygenase to prevent leukotriene synthesis (e.g. West et al., 2004). It has been reported to enhance glucose clearance and insulin sensitivity and to dramatically reduce serum triglycerides in a rat model of type II diabetes (Reed et al., 1999). The latter effect may be related in part to NDGA's reported ability to block fatty acid synthesis in adipocytes through inhibition of fatty acid synthase (FAS) and to inhibit lipoprotein lipase (Park \& Pariza, 2001; Li et al., 2005). In numerous studies, NDGA has been reported to have anticancer activity through its action as a lipoxygenase inhibitor (e.g. McDonald et al., 2001; Hoferova et al., 2004; Nony et al., 2005). It has also been reported to suppress growth of breast cancer cells through inhibition of the function of two receptor tyrosine kinases (RTK), the insulin-like growth factor receptor (IGF-R) and c-erbB2/HER2/neu (HER2/neu) (Youngren et al., 2005). NDGA may act to prevent deleterious effects of aging on the brain. Expression of 5-lipoxygenase is elevated with age and has been proposed to play a role in the pathobiology of aging-associated neurodegenerative diseases (Manev et al., 2000; Qu et al., 2000). Indeed, NDGA has been reported to be effective in preventing neuronal death and cognitive deficits resulting from forebrain ischemia-reperfusion injury (Shishido et al., 2001).

Although we do not yet know whether NDGA will extend maximum lifespan, our data show that this compound does diminish mortality risks among genetically heterogeneous, male, adult mice prior to the last third of the expected lifespan, and thus suggest that further studies of its effects on development and aging are likely to be worthwhile. Such studies might usefully include evaluations of effects of NDGA on multiple markers of health in middle-aged mice, including analyses of CNS function, T cell subsets, collagen cross-linking, lens turbidity, and early signs of neoplastic and degenerative illnesses. Evaluations of possible modes of action of NDGA, including measurements 
of hormonal, oxidative, and metabolic indices, as well as studies of patterns of gene expression and protein profiles, and the function of key tissues and organ systems, could help to test specific hypotheses about NDGA-sensitive pathways relevant to the aging process and pathogenesis of late life illnesses. Studies of other compounds with structures or pharmacologic profiles similar to NDGA could also be informative. Our data also provide a rationale for evaluation of NDGA effects on mortality risks in other varieties of mice, including other genetically heterogeneous stocks, and potentially for parallel studies using short-lived primates. Lastly, administration of NDGA to mice whose aging rate is unusually slow, for example mice on calorically restricted or methionine-restricted diets, or pituitary dwarf mutants, could help to determine whether the pathways by which NDGA affects survival overlap those involved in other genetic or nongenetic models of lifespan extension in mice.

\section{Acknowledgments}

This work was supported by NIA grants AG022303 (RAM), AG025707 and AG022308 (DEH), and AG022307 and AG13319 (RS). We wish to thank Vivian Diaz, Elizabeth Fernandez, Melissa Han, Patricia Harrison, Bill Kohler, Pam Krason, Jessica Sewald, and Maggie Vergara for technical support. We thank Scott Pletcher and Andrzej Galecki for assistance in power analysis and study design.

\section{References}

Akiyama H, Barger S, Barnum S, Bradt B, Bauer J, Cole GM, Cooper NR, Eikelenboom P, Emmerling M, Fiebich BL, Finch CE, Frautschy S, Griffin WS, Hampel H, Hull M, Landreth G, Lue L, Mrak R, Mackenzie IR, McGeer PL, O'Banion MK, Pachter J, Pasinetti G, Plata-Salaman C, Rogers J, Rydel R, Shen Y, Streit W, Strohmeyer R, Tooyoma I, Van Muiswinkel FL, Veerhuis R, Walker D, Webster S, Wegrzyniak B, Wenk G, Wyss-Coray T (2000) Inflammation and Alzheimer's disease. Neurobiol. Aging 21, 383-421.

Arteaga S, Andrade-Cetto A, Cardenas R (2005) Larrea tridentata (Creosote bush), an abundant plant of Mexican and US-American deserts and its metabolite nordihydroguaiaretic acid. J. Ethnopharmacol. 98, 231-239.

Brunelli S, Sciorati C, D'Antona G, Innocenzi A, Covarello D, Galvez BG, Perrotta C, Monopoli A, Sanvito F, Bottinelli R, Ongini E, Cossu G, Clementi E (2007) Nitric oxide release combined with nonsteroidal antiinflammatory activity prevents muscular dystrophy pathology and enhances stem cell therapy. Proc. Natl Acad. Sci. USA 104, 264269.

Buu-Hoi NP, Ratsimamanga AR (1959) [Retarding action of nordihydroguiaiaretic acid on aging in the rat]. [French]. Comptes Rendus des Seances de la Societe de Biologie et de Ses Filiales 153, 1180-1182.

Caballero F, Gerez E, Batlle A, Vazquez E (2000) Preventive aspirin treatment of streptozotocin induced diabetes: blockage of oxidative status and revertion of heme enzymes inhibition. Chem.-Biol. Interact. 126, 215-225.

Committee on Animal Models for Research on Aging. (1981) Mammalian Models for Research on Aging. Washington, DC: National Academy Press.

Denda A, Tang Q, Endoh T, Tsujiuchi T, Horiguchi K, Noguchi O, Mizumoto Y, Nakae D, Konishi Y (1994) Prevention by acetylsalicylic acid of liver cirrhosis and carcinogenesis as well as generations of 8hydroxydeoxyguanosine and thiobarbituric acid-reactive substances caused by a choline-deficient, L-amino acid-defined diet in rats. Carcinogenesis 15, 1279-1283.

Edamatsu R, Mori A, Packer L (1995) The spin-trap N-tert-alpha-phenylbutylnitrone prolongs the life span of the senescence accelerated mouse. Biochem. Biophys. Res. Commun. 211, 847-849.

Ershler WB, Keller ET (2000) Age-associated increased interleukin-6 gene expression, late-life diseases, and frailty. Annu. Rev. Med. 51, 245-270.

Ferrucci L, Harris TB, Guralnik JM, Tracy RP, Corti MC, Cohen HJ, Penninx B, Pahor M, Wallace R, Havlik RJ (1999) Serum IL-6 level and the development of disability in older persons. J. Am. Geriatr. Soc. 47, 639-646.

Floyd RA (1990) Role of oxygen free radicals in carcinogenesis and brain ischemia. FASEB J. 4, 2587-2597.

Floyd RA, Kotake Y, Hensley K, Nakae D, Konishi Y (2002) Reactive oxygen species in choline deficiency induced carcinogenesis and nitrone inhibition. Mol. Cell. Biochem. 234-235, 195-203.

Hanlon P, Lorenz WA, Shao Z, Harper JM, Galecki AT, Miller RA, Burke DT (2006) Three-locus and four-locus QTL interactions influence mouse insulin-like growth factor-I. Physiol. Genomics 26, 46-54.

Harper JM, Galecki AT, Burke DT, Miller RA (2004) Body weight, hormones and T-cell subsets as predictors of lifespan in genetically heterogeneous mice. Mech. Ageing Dev. 125, 381-390.

Harper JM, Galecki AT, Burke DT, Pinkosky SL, Miller RA (2003) Quantitative trait loci for insulin-like growth factor-I, leptin, thyroxine, and corticosterone in genetically heterogeneous mice. Physiol. Genomics $15,44-51$

Harris TB, Ferrucci L, Tracy RP, Corti MC, Wacholder S, Ettinger WH, Jr, Heimovitz H, Cohen HJ, Wallace R (1999) Associations of elevated interleukin-6 and C-reactive protein levels with mortality in the elderly. Am. J. Med. 106, 506-512.

Hoferova Z, Soucek K, Hofmanova J, Hofer M, Chramostova K, Fedorocko P, Kozubik A (2004) In vitro proliferation of fibrosarcoma cells depends on intact functions of lipoxygenases and cytochrome P-450-monooxygenase. Cancer Invest. 22, 234-247.

Holloszy JO (1993) Exercise increases average longevity of female rats despite increased food intake and no growth retardation. J. Gerontol. Biol. Sci. 48, B97-B100.

Jackson AU, Fornes A, Galecki A, Miller RA, Burke DT (1999) Multipletrait quantitative trait loci analysis using a large mouse sibship. Genetics 151, 785-795.

Jackson AU, Galecki AT, Chrisp C, Burke DT, Miller RA (2002) Mouse loci associated with life span exhibit sex-specific and epistatic effects. J. Gerontol. Biol. Sci. 57, B9-B15.

Jones RHV, Hothersall JS (1993) Increased susceptibility to metal catalysed oxidation of diabetic lens [beta]L crystallin: possible protection by dietary supplementation with acetylsalicylic acid. Exp. Eye Res. 57, 783-790.

Li BH, Ma XF, Wang Y, Tian WX (2005) Structure-activity relationship of polyphenols that inhibit Fatty Acid synthase. J. Biochem (Tokyo) 138, 679-685.

van Groen T, Kadish I (2005) Transgenic AD model mice, effects of potential anti-AD treatments on inflammation and pathology. Brain Res. Brain Res. Rev. 48, 370-378.

Manev H, Uz T, Sugaya K, Qu T (2000) Putative role of neuronal 5lipoxygenase in an aging brain. FASEB J. 14, 1464-1469.

Masferrer JL, Leahy KM, Koki AT, Zweifel BS, Settle SL, Woerner BM, Edwards DA, Flickinger AG, Moore RJ, Seibert K (2000) Antiangiogenic and antitumor activities of cyclooxygenase-2 inhibitors. Cancer Res. 60, 1306-1311.

McDonald RW, Bunjobpon W, Liu T, Fessler S, Pardo OE, Freer IK, Glaser M, Seckl MJ, Robins DJ (2001) Synthesis and anticancer activity of nordihydroguaiaretic acid (NDGA) and analogues. Anticancer Drug Des. 16, 261-270. 
Miller RA (2002) Extending life: scientific prospects and political obstacles. Milbank Q. 80, 155-174.

Miller RA, Austad S, Burke D, Chrisp C, Dysko R, Galecki A, Monnier V (1999a) Exotic mice as models for aging research: polemic and prospectus. Neurobiol. Aging 20, 217-231.

Miller RA, Burke D, Nadon N (1999b) Announcement: four-way cross mouse stocks: a new, genetically heterogeneous resource for aging research. J. Gerontol. A Biol. Sci. Med. Sci. 54A, B358.

Miller RA, Chrisp C (1999) Lifelong treatment with oral DHEA sulfate does not preserve immune function, prevent disease, or improve survival in genetically heterogeneous mice. J. Am. Geriatr. Soc. 47, 960-966.

Miquel J, Fleming J, Economos AC (1982) Antioxidants, metabolic rate and aging in Drosophila. Arch. Gerontol. Geriatr. 1, 159-165.

Munkres KD, Colvin HJ (1976) Ageing of Neurospora crassa. II. Organic hydroperoxide toxicity and the protective role of antioxidant and the antioxygenic enzymes. Mech. Ageing Dev. 5, 99-107.

Nakae D, Kishida H, Enami T, Konishi Y, Hensley KL, Floyd RA, Kotake Y (2003) Effects of phenyl N-tert-butyl nitrone and its derivatives on the early phase of hepatocarcinogenesis in rats fed a choline-deficient, 1-amino acid-defined diet. Cancer Sci. 94, 26-31.

Nony PA, Kennett SB, Glasgow WC, Olden K, Roberts JD (2005) 15SLipoxygenase-2 mediates arachidonic acid-stimulated adhesion of human breast carcinoma cells through the activation of TAK1, MKK6, and p38 MAPK. J. Bio.I Chem. 280, 31413-31419.

Olshansky SJ, Carnes BA, Cassel C (1990) In search of Methuselah: estimating the upper limits to human longevity. Science 250, 634-640.

Olshansky SJ, Perry D, Miller RA, Butler RN (2006) In pursuit of the longevity dividend. Scientist 20, 28-35.

Ongini E, Bolla M (2006) Nitric-oxide based nonsteroidal anti-inflammatory agents. Drug Discovery Today: Therapeutic Strategies 3, 395-400.

Pincus T (2001) Clinical evidence for osteoarthritis as an inflammatory disease. Curr. Rheumatol. Rep. 3, 524-534.

Qu T, Uz T, Manev H (2000) Inflammatory 5-LOX mRNA and protein are increased in brain of aging rats. Neurobiol. Aging 21, 647-652.

Reed MJ, Meszaros K, Entes L, Claypool MD, Pinkett JG, Brignetti D, Luo J, Khandwala A, Reaven GM (1999) Effect of masoprocol on carbohydrate and lipid metabolism in a rat model of Type II diabetes. Diabetologia. 42, 102-106.

Richie JP, Jr, Mills BJ, Lang CA (1986) Dietary nordihydroguaiaretic acid increases the life span of the mosquito. Proc. Soc. Exp. Biol. Med. $183,81-85$.

Sack CA, Socci DJ, Crandall BM, Arendash GW (1996) Antioxidant treatment with phenyl-alpha-tert-butyl nitrone (PBN) improves the cognitive performance and survival of aging rats. Neurosci. Lett. 205, 181-184.

Saito K, Yoshioka H, Cutler RG (1998) A spin trap, N-tert-butyl-alphaphenylnitrone extends the life span of mice. Biosci. Biotechnol. Biochem. 62, 792-794.

Schneider EL, Miller RA (1998) Anti-aging interventions. In Brockelhurst's Textbook of Geriatric Medicine (Tallis R, Fillit H, Brockelhurst JC, eds). New York: Churchill Livingstone, pp. 193-199.
Schneider EL, Reed JD, Jr (1985) Life extension. N. Engl. J. Med. 312, $1159-1168$.

Shackelford RE, Alford PB, Xue Y, Thai SF, Adams DO, Pizzo S (1997) Aspirin inhibits tumor necrosis factor- $\alpha$ gene expression in murine tissue macrophages. Mol. Pharmacol. 52, 421-429.

Shi X, Min D, Zigang D, Fei C, Jiangping Y, Suwei W, Stephen S, Leonard Vince C, Val V (1999) Antioxidant properties of aspirin: characterization of the ability of aspirin to inhibit silica-induced lipid peroxidation, DNA damage, NF- $\kappa \mathrm{B}$ activation, and TNF- $\alpha$ production. Mol. Cell. Biochem. V199, 93-102.

Shishido Y, Furushiro M, Hashimoto S, Yokokura T (2001) Effect of nordihydroguaiaretic acid on behavioral impairment and neuronal cell death after forebrain ischemia. Pharmacol Biochem Behav. 69, 469474.

Spaulding CC, Walford RL, Effros RB (1997) Calorie restriction inhibits the age-related dysregulation of the cytokines TNF- $\alpha$ and IL- 6 in C3B10RF1 mice. Mech. Ageing Dev. 93, 87-94.

Vane SJ (2000) Aspirin and other anti-inflammatory drugs. Thorax 55, 3S-9.

Wang C, Li Q, Redden DT, Weindruch R, Allison DB (2004) Statistical methods for testing effects on 'maximum lifespan'. Mech. Ageing Dev. 125, 629-632.

Warner HR, Ingram D, Miller RA, Nadon NL, Richardson AG (2000) Program for testing biological interventions to promote healthy aging. Mech. Ageing Dev. 115, 199-207.

Weber C, Erl W, Pietsch A, Weber PC (1995) Aspirin inhibits nuclear factor $\kappa B$ mobilization and monocyte adhesion in stimulated human endothelial cells. Circulation 91, 1914-1917.

Weissberg PL, Bennett MR (1999) Atherosclerosis - an inflammatory disease. N. Engl. J. Med. 340, 1928-1929.

Weissmann G (1991) Aspirin. Sci. Am. 264, 84-90.

West M, Mhatre M, Ceballos A, Floyd RA, Grammas P, Gabbita SP, Hamdheydari L, Mai T, Mou S, Pye QN, Stewart C, West S, Williamson KS, Zemlan F, Hensley K (2004) The arachidonic acid 5-lipoxygenase inhibitor nordihydroguaiaretic acid inhibits tumor necrosis factor alpha activation of microglia and extends survival of G93A-SOD1 transgenic mice. J. Neurochem. 91, 133-143.

Wood JG, Rogina B, Lavu S, Howitz K, Helfand SL, Tatar M, Sinclair D (2004) Sirtuin activators mimic caloric restriction and delay ageing in metazoans. [erratum appears in Nature 2004 Sep 2;431(7004):107]. Nature 430, 686-689.

Youngren JF, Gable K, Penaranda C, Maddux BA, Zavodovskaya M, Lobo M, Campbell M, Kerner J, Goldfine ID (2005) Nordihydroguaiaretic acid (NDGA) inhibits the IGF-1 and c-erbB2/HER2/neu receptors and suppresses growth in breast cancer cells. Breast Cancer Res Treat. 94, 37-46.

Zhao Q, Pahlmark K, Smith ML, Siesjo BK (1994) Delayed treatment with the spin trap alpha-phenyl-N-tert-butyl nitrone (PBN) reduces infarct size following transient middle cerebral artery occlusion in rats. Acta Physiol. Scand. 152, 349-350. 